\title{
Kinematic generation of Darboux cyclides
}

\author{
Niels Lubbes, Josef Schicho
}

March 15, 2019

\begin{abstract}
We state a relation between two families of lines that cover a quadric surface in the Study quadric and two families of circles that cover a Darboux cyclide.
\end{abstract}

Keywords: Study quadric, Darboux cyclides

\section{Introduction}

The Study quadric is a projective compactification of the group of Euclidean displacements. If we fix a point in 3-space, then projective varieties inside the Study quadric — considered as sets of displacements - give rise to orbit varieties in 3-space.

Let us consider the relation between a class of varieties in the Study quadric and their orbits in 3-space. A classical example is presented by the class of lines in the Study quadric; the orbit of a line is either a circle, line or point in 3-space (see Lemma 1). The orbit of a conic in the Study quadric is a rational quartic curve with full cyclicity [10]. The case of rational curves of arbitrary degree in the Study quadric is studied in [4]: a general rational curve of degree $d$ in the Study quadric has an orbit of degree $2 d$. We also have a uniqueness result: for any rational curve of degree $d$ and cyclicity $2 c$, there is a unique rational curve in the Study quadric of degree $d-c$ in the Study quadric defining that orbit [7, Theorem 2]. 
In this paper we show that the orbit of a doubly ruled quadric surface in the Study quadric is a Darboux cyclide. Darboux cyclides are surfaces that contain at least two and at most six circles through each point. These surfaces have been recently studied in $[5,6,8,9]$. Theorem 1 is again a uniqueness result: for two families of circles that cover a Darboux cyclide there exists a unique doubly ruled quadric surface in the Study quadric.

\section{The orbit map}

The dual quaternions are defined as the noncommutative associative algebra

$\mathbb{D H}:=\mathbb{R}[\mathbf{i}, \mathbf{j}, \mathbf{k}, \epsilon] /\left\langle\mathbf{i}^{2}+1, \mathbf{j}^{2}+1, \mathbf{k}^{2}+1, \mathbf{i j} \mathbf{k}+1, \epsilon^{2}, \epsilon \mathbf{i}-\mathbf{i} \epsilon, \epsilon \mathbf{j}-\mathbf{j} \epsilon, \epsilon \mathbf{k}-\mathbf{k} \epsilon\right\rangle$

We consider the following coordinates for $h \in \mathbb{D H}$ and $\bar{h} \in \mathbb{D H}$ :

$$
\begin{aligned}
& h=p+q \epsilon=\left(p_{0}+p_{1} \mathbf{i}+p_{2} \mathbf{j}+p_{3} \mathbf{k}\right)+\left(q_{4}+q_{5} \mathbf{i}+q_{6} \mathbf{j}+q_{7} \mathbf{k}\right) \epsilon \\
& \bar{h}=\bar{p}+\bar{q} \epsilon=\left(p_{0}-p_{1} \mathbf{i}-p_{2} \mathbf{j}-p_{3} \mathbf{k}\right)+\left(q_{4}-q_{5} \mathbf{i}-q_{6} \mathbf{j}-q_{7} \mathbf{k}\right) \epsilon
\end{aligned}
$$

We denote by $N: \mathbb{D H} \rightarrow \mathbb{D}, h \mapsto h \bar{h}$, the dual quaternion norm. By projectivizing $\mathbb{D H}$ as a real 8 -dimensional vector space, we obtain $\mathbb{P}^{7}$. The Study quadric is defined as

$$
S:=\left\{h \in \mathbb{P}^{7} \mid h \bar{h} \in \mathbb{R}\right\}=\left\{p+q \epsilon \in \mathbb{P}^{7} \mid p_{0} q_{0}+p_{1} q_{1}+p_{2} q_{2}+p_{3} q_{3}=0\right\} .
$$

The Study boundary $B \subset S$ is defined as $B:=\{h \in S \mid h \bar{h}=0\}$. If we identify $\mathbb{R}^{3}$ with $\left\{v \in \mathbb{D H} \mid v=v_{1} \mathbf{i}+v_{2} \mathbf{j}+v_{3} \mathbf{k}\right\}$, then the Study kinematic mapping is a group action

$$
\varphi:(S \backslash B) \times \mathbb{R}^{3} \rightarrow \mathbb{R}^{3}, \quad(p+q \epsilon, v) \mapsto \frac{p v \bar{p}+p \bar{q}-q \bar{p}}{p \bar{p}},
$$

and $S \backslash B \cong S E(3)$ via this action [3, Section 2.1]. We choose the following coordinates for the 3-dimensional Möbius quadric:

$$
\mathbb{S}^{3}:=\left\{x \in \mathbb{P}^{4} \mid x_{0} x_{4}-x_{1}^{2}-x_{2}^{2}-x_{3}^{2}=0\right\} .
$$


With this somewhat unusual choice of coordinates the stereographic projection with center $(0: 0: 0: 0: 1) \in \mathbb{S}^{3}$ is defined as

$$
\tau: \mathbb{S}^{3} \rightarrow \mathbb{P}^{3}, \quad\left(x_{0}: \ldots: x_{4}\right) \mapsto\left(x_{0}: x_{1}: x_{2}: x_{3}\right)
$$

For any point $u=\left(u_{0}: \ldots: u_{4}\right) \in \mathbb{S}^{3}$ such that $u_{0} \neq 0$, the orbit map is defined as

$$
\begin{aligned}
\operatorname{orb}_{u}: S \backslash F_{u} & \rightarrow \mathbb{S}^{3} \\
p+q \epsilon & \mapsto\left(p \bar{p}: w_{1}: w_{2}: w_{3}: 4 q \bar{q}-p \bar{p} v^{2}+2(q v \bar{p}-p v \bar{q})\right),
\end{aligned}
$$

where $p v \bar{p}+p \bar{q}-q \bar{p}=w_{1} \mathbf{i}+w_{2} \mathbf{j}+w_{3} \mathbf{k}$ with $v=\frac{u_{1}}{u_{0}} \mathbf{i}+\frac{u_{2}}{u_{0}} \mathbf{j}+\frac{u_{3}}{u_{0}} \mathbf{k}$ the dehomogenization of $\tau(u)$ and

$$
F_{u}:=\{p+q \epsilon \in S \mid p \bar{p}=p v \bar{p}+p \bar{q}-q \bar{p}=4 q \bar{q}+2(q v \bar{p}-p v \bar{q})=0\} \subset B
$$

Notice that the orbit map is the composition of the projective closure of $\varphi(\cdot, v): S \rightarrow \mathbb{R}^{3}$ with the inverse stereographic projection. We fix notation for the identity $\mathfrak{e}:=(1: 0: \ldots: 0) \in S$ and the origin $\mathfrak{o}:=(1: 0: \ldots: 0) \in$ $\mathbb{S}^{3}$.

Proposition 1. The Zariski closure of the image of orb $_{u}$ is $\mathbb{S}^{3}$ and $F_{u} \subset S$ is a quartic 4-fold.

Proof. Suppose that $p+\epsilon q \in S$ so that $p \bar{q}+q \bar{p}=0$ and $w:=p v \bar{p}+p \bar{q}-q \bar{p}$. We find that $w \bar{w}=-(p v \bar{p}+2 p \bar{q})^{2}=p \bar{p} \cdot\left(4 q \bar{q}-p \bar{p} v^{2}+2(q v \bar{p}-p v \bar{q})\right)$. It follows that the image of $\operatorname{orb}_{u}$ is contained in $\mathbb{S}^{3}$, since $w \bar{w}=w_{1}^{2}+w_{2}^{2}+w_{3}^{2}$. Recall that $S \backslash B$ is isomorphic to $S E(3)$ via the Study kinematic mapping, and thus the image of $\operatorname{orb}_{u}$ is Zariski dense in $\mathbb{S}^{3}$.

An Euclidean isometry of $\mathbb{R}^{3}$ is realized by an automorphism of $S$ that preserves $B$. Thus there exists $e \in S \backslash B$ such that $\operatorname{orb}_{\mathfrak{o}}(e)=u$. Since the automorphisms of $S \backslash B$ and $\mathbb{R}^{3}$ are transitive, we may assume without loss of generality that $e=\mathfrak{e}$ and $u=\mathfrak{o}$. The locus where orb $_{\mathfrak{o}}$ is not defined equals

$$
F_{\mathfrak{o}}=\{p+q \epsilon \in \mathbb{D} \mathbb{H} \mid p \bar{q}=q \bar{p}=p \bar{p}=q \bar{q}=0\} \subset S .
$$


We computed with a computer algebra system the Hilbert function of the ideal of $F_{\mathfrak{o}}$ and find that $\operatorname{dim} F_{\mathfrak{o}}=\operatorname{deg} F_{\mathfrak{o}}=4$. This concludes the proof of this proposition.

A circle is an irreducible conic in $\mathbb{S}^{3}$. Let

$$
\begin{aligned}
& \mathcal{L}_{\mathfrak{e}}:=\{\ell \subset S \mid \ell \text { is a line such that } \mathfrak{e} \in \ell\}, \\
& \mathcal{C}_{\mathfrak{o}}:=\left\{C \subset \mathbb{S}^{3} \mid C \text { is either a circle or a point such that } \mathfrak{o} \in C\right\} .
\end{aligned}
$$

Lemma 1. If $\ell \subset S$ is a line, then $\operatorname{orb}_{u}(\ell)$ is a circle or a point. Moreover, the following map is almost everywhere one-to-one

$$
\psi: \mathcal{L}_{\mathfrak{e}} \rightarrow \mathcal{C}_{\mathfrak{o}}, \quad \ell \mapsto \operatorname{orb}_{\mathfrak{o}}(\ell)
$$

Proof. Lines in the Study quadric correspond to either rotations or translations [3, Section 2.5] and orbits under these 1-parameter subgroups are circles, lines or points in $\mathbb{R}^{3}$ und thus via the stereographic projecion $\tau$ points or circles in $\mathbb{S}^{3}$.

We can associate to a circle in the 4-dimensional set $\mathcal{C}_{\mathfrak{o}}$, a unique 1-parameter subgroup of rotations corresponding to a line in $\mathcal{L}_{\mathfrak{e}}$. There is a 2-dimensional set of lines in $\mathcal{L}_{\mathfrak{e}}$ such that the rotational axis of these lines passes through $\mathfrak{o}$ and the corresponding Lie circle is the point $\mathfrak{o}$. Thus $\psi$ is one-to-one except for a lower dimensional subset as was claimed.

\section{Quadric surfaces in the Study quadric}

A Darboux cyclide is defined as a quartic weak del Pezzo surface in $\mathbb{S}^{3}[2$, Section 8.6.2]. Such surfaces are the intersection of $\mathbb{S}^{3}$ with a quadric hypersurface [2, Theorem 8.6.2]. Let $\mathcal{U}_{\mathfrak{o}}$ denote the set of quadric surfaces $Q \subset S$ such that either there exists $V \cong \mathbb{P}^{3}$ such that $Q \subset V \subset S$, or $Q \subset F_{\mathfrak{o}} \subset S$.

Lemma 2. If $Q \subset S$ is a doubly ruled quadric surface such that $Q \notin \mathcal{U}_{\mathfrak{o}}$, then $\operatorname{orb}_{\mathfrak{o}}(Q) \subset \mathbb{S}^{3}$ is a Darboux cyclide. 
Proof. There exists bilinear homogeneous $a+\epsilon b \in \mathbb{D} \mathbb{H}\left[s_{0}, s_{1}, t_{0}, t_{1}\right]$ such that $Q$ is parametrized by

$$
\mu: \mathbb{P}^{1} \times \mathbb{P}^{1} \rightarrow Q \subset S, \quad\left(s_{0}: s_{1} ; t_{0}: t_{1}\right) \mapsto a+\epsilon b,
$$

and $a \bar{b}-b \bar{a}=w_{1} \mathbf{i}+w_{2} \mathbf{j}+w_{3} \mathbf{k}$ with $w_{1}, w_{2}, w_{3} \in \mathbb{R}\left[s_{0}, s_{1}, t_{0}, t_{1}\right]$ so that

$$
\begin{aligned}
\operatorname{orb}_{\mathfrak{o}} \circ \mu: \quad \mathbb{P}^{1} \times \mathbb{P}^{1} & \rightarrow D \subset \mathbb{S}^{3}, \\
(s, t) & \mapsto\left(a \bar{a}: w_{1}: w_{2}: w_{3}: 4 b \bar{b}\right) .
\end{aligned}
$$

Thus the map orb $b_{\mathfrak{o}} \circ \mu$ is of bidegree $(2,2)$ into $\mathbb{P}^{4}$. By Proposition 1 , the map $\operatorname{orb}_{\mathfrak{o}}$ is not defined at a quartic 4-fold $F_{\mathfrak{o}} \subset S$. We observe that $Q$ is the intersection of $S$ with a 3 -space, since $Q \notin \mathcal{U}_{\mathfrak{o}}$. It follows that $Q \cap F_{\mathfrak{o}}$ consists of 4 points (counted with multiplicity). Thus $\operatorname{orb}_{\mathfrak{o}} \circ \mu$ has 4 base points. A basis of all bidegree $(2,2)$ functions on $\mathbb{P}^{1} \times \mathbb{P}^{1}$ defines a map whose image is a degree 8 weak del Pezzo surface $X \subset \mathbb{P}^{8}$. A basis of bidegree $(2,2)$ functions that pass through a basepoint, defines a map whose image is a projection of $X$ from a point so that the degree and embedding dimension drops by one. Such a projection realizes the blowup of $X$ in a point and is again a weak del Pezzo surface [2, Proposition 8.1.23]. Thus $M$ can be obtained as 4 subsequent projections of $X$, which results in a quartic weak del Pezzo surface in $\mathbb{P}^{4}$. This concludes the proof of this lemma, since the image of orb $_{\mathfrak{o}}$ is $\mathbb{S}^{3}$ by Proposition 1 .

A family of curves of a surface $X$ is defined as an irreducible hypersurface $F \subset X \times \mathbb{P}^{1}$ such that the closure of the first projection of $F$ equals $X$. A
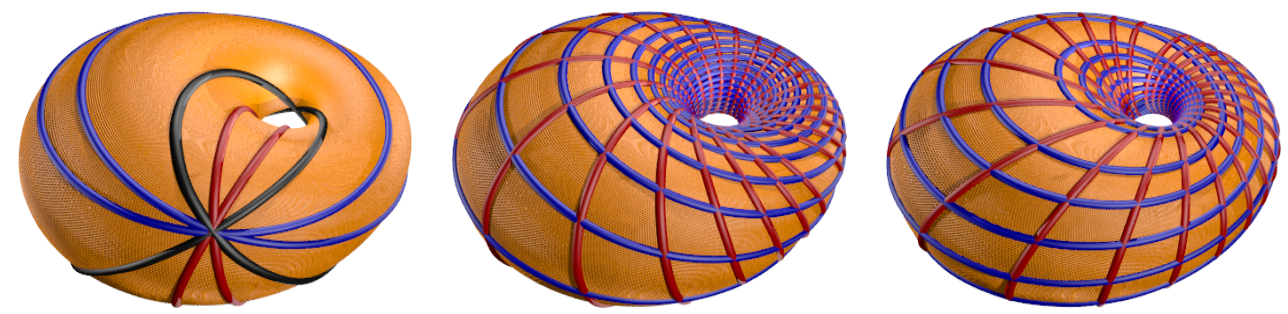

Figure 1: A smooth Darboux cyclide contains six circles through each point and admits $\left(\begin{array}{l}6 \\ 2\end{array}\right)-3$ pairs of families of circles $F$ and $F^{\prime}$ so that $F \cdot F^{\prime}=1$. 
curve $F_{t} \subset X$ in the family $F$ for some $t \in \mathbb{P}^{1}$ is defined as $\pi_{1}(F \cap X \times\{t\})$. If $F$ and $F^{\prime}$ are families of $X$, then we denote by $F \cdot F^{\prime}$ the number of intersections of a general curve in $F$ and a general curve in $F^{\prime}$. See also Figure 1.

Lemma 3. If two different Darboux cyclides in $\mathbb{S}^{3}$ intersect in three circles, then two of these circles are co-spherical.

Proof. Suppose that $D, D^{\prime} \subset \mathbb{S}^{3}$ are Darboux cyclides. We can associate to the weak del Pezzo surface $D$ its Picard group, which is a quadratic lattice $\left\langle\alpha_{0}, \alpha_{1}, \ldots, \alpha_{5}\right\rangle_{\mathbb{Z}}$ with intersection pairing $\alpha_{0}^{2}=1, \alpha_{i}^{2}=-1$ for $i>0$ and $\alpha_{i} \cdot \alpha_{j}=0$ for $i \neq j[2$, Section 8.2.1]. We associate to a curve $C \subset D$ its divisor class $[C]$ in the Picard group of $D$. The class of any hyperplane section of $D$ is equal to the anticanonical class $-\kappa=3 \alpha_{0}-\alpha_{1}-\ldots-\alpha_{5}$. Both $D$ and $D^{\prime}$ are intersections of $\mathbb{S}^{3}$ with a quadric hypersurface so that $\left[D \cap D^{\prime}\right]=-2 \kappa$ and $\operatorname{deg} D \cap D^{\prime}=8$. Since $D \cap D^{\prime}$ contains 3 circles by assumption, it follows that $D \cap D^{\prime}$ consists of 4 conics. The class of a conic in $M$ is either $\alpha_{0}-\alpha_{i}$ or $2 \alpha_{0}+\alpha_{i}-\alpha_{1}-\ldots-\alpha_{5}$ for some $1 \leq i \leq 5$. The classes of the conics have to add up to $-2 \kappa$, and must be of the following form

$\left(\alpha_{0}-\alpha_{i}\right)+\left(2 \alpha_{0}+\alpha_{i}-\alpha_{1}-\ldots-\alpha_{5}\right)+\left(\alpha_{0}-\alpha_{j}\right)+\left(2 \alpha_{0}+\alpha_{j}-\alpha_{1}-\ldots-\alpha_{5}\right)$,

for some $1 \leq i, j \leq 5$. Since $\left(\alpha_{0}-\alpha_{i}\right) \cdot\left(2 \alpha_{0}+\alpha_{i}-\alpha_{1}-\ldots-\alpha_{5}\right)=2$ there are two co-spherical circles.

Lemma 4. If $F, F^{\prime} \subset D \times \mathbb{P}^{1}$ are families of circles on a Darboux cyclide $D \subset \mathbb{S}^{3}$ such that $F \cdot F^{\prime}=1$ and $\mathfrak{o} \in D$, then there exists a unique doubly ruled quadric surface $Q \subset S$ such that $\mathfrak{e} \in Q, Q \notin \mathcal{U}_{\mathfrak{o}}$, $\operatorname{orb}_{\mathfrak{o}}(Q)=D$ and the two rulings of $Q$ correspond via orb $\mathrm{b}_{\mathfrak{o}}$ to $F$ and $F^{\prime}$.

Proof. Let $C, C^{\prime} \subset D$ be two circles in $F$ and $F^{\prime}$ respectively, such that $C \cap C^{\prime}=\mathfrak{o}$. By Lemma 1 there exist unique lines $\ell, \ell^{\prime} \subset S$ containing $\mathfrak{e}$ such that $\operatorname{orb}_{\mathfrak{o}}(\ell)=C$ and $\operatorname{orb}_{\mathfrak{o}}\left(\ell^{\prime}\right)=C^{\prime}$. We choose some point on $h \in \ell^{\prime}$ and 
let $C^{\prime \prime}$ be the unique circle in the family $F$ that passes through $p:=\operatorname{orb}_{\mathfrak{o}}(h)$. We apply Lemma 1 with $\mathfrak{o}$ replaced by $p$ and obtain a unique line $L \subset S$ containing $\mathfrak{e}$. It follows from the construction that $C^{\prime \prime}=\operatorname{orb}_{\mathfrak{o}}\left(\ell^{\prime \prime}\right)$, where $\ell^{\prime \prime}:=h L$ and $h L$ means the image of each point in $L$ multiplied with the dual quaternion $h$.
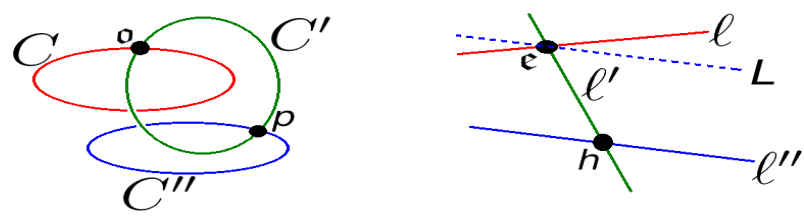

Thus we obtain three intersecting lines $\ell, \ell^{\prime}, \ell^{\prime \prime} \subset S$ spanning a 3 -space $V$ and three circles $C, C^{\prime}, C^{\prime \prime} \subset D$ that pairwise intersect in at most one point. We have that $V \nsubseteq S$, otherwise $\operatorname{orb}_{\mathfrak{o}}(V)$ would be either a point or a 2-sphere by Lemma 1. Thus $V \cap S$ defines a unique quadric surface $Q \subset S$ such that $\ell, \ell^{\prime}, \ell^{\prime \prime} \subset Q$ and $Q \notin \mathcal{U}_{\mathfrak{o}}$. It follows from Lemma 2 that $D^{\prime}:=\operatorname{orb}_{\mathfrak{o}}(Q)$ is a Darboux cyclide such that $C, C^{\prime}, C^{\prime \prime} \subset D \cap D^{\prime}$. It follows from Lemma 3 that $D$ and $D^{\prime}$ must be equal. By Lemma 1 , lines in $S$ correspond to circles in $\mathbb{S}^{3}$ and a quadric $Q \subset S$ is covered by two families of lines. This concludes the proof of this lemma.

Remark 1. For the existence statement in Lemma 4, it is also possible to give an algebraic proof without using Lemma 3. By [11, Theorem 11], there exists a parametrization for Darboux cyclides of bidegree $(2,2)$ so that the parameter curves are the circles in $F_{1}$ and $F_{2}$, respectively. Lifting the parametrization to $\mathbb{S}^{3}$, we obtain 5 biquadratic polynomials $X_{0}, \ldots, X_{5} \in$ $\mathbb{R}[s, t]$ such that $X_{0} X_{4}=X_{1}^{2}+X_{2}^{2}+X_{3}^{2}$ and $\left(X_{0}: X_{1}: X_{2}: X_{3}: X_{4}\right)$ is a parametrization of the Darboux cyclide $D$. By [6, Theorem 3], there exist bilinear polynomials $A, B \in \mathbb{H}[s, t]$ with quaternion coefficients such that

$$
\mathrm{N}(A)=X_{0}, \quad \mathrm{~N}(B)=X_{4}, \quad A B=X_{1} \mathbf{i}+X_{2} \mathbf{j}+X_{3} \mathbf{k} .
$$

The bilinear polynomial $H:=\bar{A}+\epsilon B \in \mathbb{D} \mathbb{H}[s, t]$ then defines a parametrization of a nonsingular ruled quadric in the Study quadric $S$, and the image of this quadric via orb $b_{\mathfrak{o}}$ is exactly $D$. 
Theorem 1. The map orb $: S \backslash F_{\mathfrak{o}} \rightarrow \mathbb{S}^{3}$ defines a one-to-one correspondence between two families of lines that cover a quadric surface $Q \subset S$ such that $\mathfrak{e} \in Q$ with $Q \notin \mathcal{U}_{0}$ - and - two non-cospherical families of circles that cover a Darboux cyclide $D \subset \mathbb{S}^{3}$ such that $\mathfrak{o} \in D$.

Proof. The left to right direction is a consequence of Lemma 2 and Lemma 1. The converse direction follows from Lemma 4.

For example, let $D \subset \mathbb{S}^{3}$ be a surface that is covered by exactly 6 families of circles and suppose that $\mathfrak{o} \in D$. In this case $D$ is a Darboux cyclide and admits 15 pairs of families of circles [1,9]. There are exactly three pairs $\left(F, F^{\prime}\right)$ of families of circles such that $F \cdot F^{\prime}=2$ (see Figure 1). For the remaining 12 pairs $\left(F, F^{\prime}\right)$ of families one has $F \cdot F^{\prime}=1$ and thus by Theorem 1 there are 12 quadric surfaces $Q \subset S$ such that $\mathfrak{e} \in Q$ and $\operatorname{orb}_{\mathfrak{o}}(Q)=D$.

\section{Acknowledgements}

We thank R. Krasauskas for the algebraic proof of the existence statement in Lemma 4 (see Remark 1) and M. Skopenkov for the help in the analysis of the algebraic statements underlying this proof.

\section{References}

[1] R. Blum. Circles on surfaces in the Euclidean 3-space. In Geometry and differential geometry (Proc. Conf., Univ. Haifa, Haifa, 1979), volume 792 of Lecture Notes in Math., pages 213-221. Springer, Berlin, 1980.

[2] I. V. Dolgachev. Classical algebraic geometry: A modern view. Cambridge University Press, Cambridge, 2012. 
[3] M. Husty and H.-P. Schröcker. Algebraic geometry and kinematics. In Nonlinear computational geometry, volume 151 of IMA Vol. Math. Appl., pages 85-107. Springer, 2010.

[4] B. Jüttler. Über zwangläufige rationale Bewegungsvorgänge. Österreich. Akad. Wiss. Math.-Natur. Kl. Sitzungsber. II, 202(1-10):117-132, 1993.

[5] R. Krasauskas and M. Skopenkov. Surfaces containing two circles through each point and Pythagorean 6-tuples. arXiv:1503.06481v2, 2015.

[6] R. Krasauskas and S. Zube. Rational bezier formulas with quaternion and clifford algebra weights. SAGA - Advances in ShApes, Geometry, and Algebra, Geometry and Computing, 10:147-166, 2014.

[7] Z. Li, J. Schicho, and H.-P. Schröcker. The rational motion of minimal dual quaternion degree with prescribed trajectory. Comput. Aided Geom. Design, 41:1-9, 2016.

[8] M. Peternell. Generalized dupin cyclides with rational lines of curvature. Lecture Notes in Computer Science, 6920:543-552, 2012.

[9] H. Pottmann, L. Shi, and M. Skopenkov. Darboux cyclides and webs from circles. Comput. Aided Geom. Design, 29(1):77-97, 2012.

[10] O. Röschel. Rationale räumliche Zwangläufe vierter Ordnung. Österreich. Akad. Wiss. Math.-Natur. Kl. Sitzungsber. II, 194(4-10): 185-202, 1985.

[11] J. Schicho. The multiple conical surfaces. Beitr. Alg. Geom., 42:71-87, 2001.

N. Lubbes, Johann Radon Institute for Computational and Applied Mathematics (RICAM), Austrian Academy of Sciences

email: niels.lubbes@gmail.com

J. Schicho, Research Institute for Symbolic Computation (RISC), Johannes Kepler University

email: josef.schicho@risc.jku.at 